\title{
Improvement of electron beam quality in optical injection schemes using negative plasma density gradients
}

\author{
G. Fubiani, * E. Esarey, C. B. Schroeder, and W. P. Leemans \\ Lawrence Berkeley National Laboratory, \\ University of California, Berkeley, CA 94720
}

(Dated: July 26, 2005)

\begin{abstract}
Enhanced electron trapping using plasma density down ramps as a method for improving the performance of laser injection schemes is proposed and analyzed. A decrease in density implies an increase in plasma wavelength, which can shift a relativistic electron from the defocusing to the focusing region of the accelerating wakefield, and a decrease in wake phase velocity, which lowers the trapping threshold. The specific method of two-pulse colliding pulse injector was examined using a three-dimensional test particle tracking code. A density down-ramp with a change of density on the order of tens of percent over distances greater than the plasma wavelength led to an enhancement of charge by two orders in magnitude or more, up to the limits imposed by beam loading. The accelerated bunches are ultrashort (fraction of the plasma wavelength, e.g., $\sim 5 \mathrm{fs}$ ), high charge $\left(>20 \mathrm{pC}\right.$ at modest injection laser intensity $\left.\sim 10^{17} \mathrm{~W} / \mathrm{cm}^{2}\right)$, with a relative energy spread of a few percent at a mean energy of $\sim 25 \mathrm{MeV}$, and a normalized root-mean square emittance on the order $0.5 \mathrm{~mm}$ mrad.
\end{abstract}

PACS numbers: 52.38.Kd

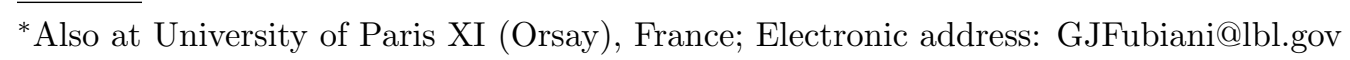




\section{INTRODUCTION}

Compared to standard radio-frequency $(\mathrm{RF})$ linear accelerators, advanced accelerators using plasmas can produce much higher acceleration gradients, in excess of $10 \mathrm{GeV} / \mathrm{m}$ without the limitation of breakdown. In a plasma, the wavelength of the acceleration field is the plasma wavelength, $\lambda_{p}=2 \pi c / \omega_{p}$, or $\lambda_{p}[\mathrm{~m}] \simeq 3.3 \times 10^{4}\left(n_{0}\left[\mathrm{~cm}^{-3}\right]\right)^{-1 / 2}$, where $n_{0}$ is the plasma density, $c$ is the speed of light, $\omega_{p}=\left(4 \pi n_{0} e^{2} / m_{e}\right)^{1 / 2}$ is the plasma frequency, $m_{e}$ the electron mass and $-e$ the electron charge. For example, a laser wakefield accelerator (LWFA) [1] in the standard regime typically has a density on the order of $n_{0} \simeq 10^{18} \mathrm{~cm}^{-3}$ and a plasma wavelength on the order of $\lambda_{p} \simeq 30 \mu \mathrm{m}$. If a mono-energetic electron bunch is injected into a wakefield such that it is accelerated while maintaining a small energy spread, then it is necessary for the bunch to occupy a small fraction of the wake period, on the order of a few femtoseconds. This requires truly femtosecond accuracy in the injection process, which is beyond the current state-of-the-art performance of photocathode radio-frequency electron guns.

Several injection mechanisms of plasma electrons into the accelerating wake have been described that rely on self-trapping. In a homogeneous plasma, self-trapping can occur by driving the wake to the wavebreaking limit in the self-modulated LWFA regime $[2,3]$, in the highly-nonlinear blow-out or bubble regime [4, 5], or in the two-dimensional wavebreaking regime [6]. In an inhomogeneous plasma, a gradual density down-ramp will eventually lead to wavebreaking some distance behind the drive beam, due to decrease in the wake phase velocity on the ramp [7, 8]. Alternatively, a strong drive pulse can lead to trapping at a sudden discontinuity in the plasma density [9].

In an effort to improve the trapped bunch quality over single-beam methods, several injection methods have been proposed that utilize additional injection laser pulses. The motivation behind using additional injection pulses is to have more control over the injection process, provided the drive pulse does not create a wake of sufficient amplitude to selftrap background plasma electrons (so called dark current free powering of plasmas has recently been observed in a channel guided LWFA [10]). The injection pulse can be used to turn on and off the injection process, e.g., injection only occurs when the injection pulse intersects with the wake. The ponderomotive force associated with the envelope of a single injection laser pulse can be used to boost the electron momentum and phase such that they 
become trapped in the wakefield $[11,12,13]$. Typically, ponderomotive injection methods require high intensities $I>10^{18} \mathrm{~W} / \mathrm{cm}^{2}$ (corresponding to a normalized laser strength $\left.a \simeq 8.6 \times 10^{-10} \lambda[\mu \mathrm{m}] I^{1 / 2}\left[\mathrm{~W} / \mathrm{cm}^{2}\right]>1\right)$ in both the pump and injection laser pulses.

Alternatively, the slow phase velocity beat wave (interference term) produced by the collision of two counter-propagating (or intersecting at an angle) lasers can be used in either a three-pulse $[14,15]$ or two-pulse $[16,17,18,19]$ configuration. In the original colliding pulse injector (CPI) concept, three short laser pulses were used for electron injection $[14,15]$. The pump pulse generates a plasma wake through its ponderomotive force, as in the standard laser wakefield accelerator (LWFA). The two injection lasers, one pulse propagating in the forward direction behind the pump laser pulse and the other in the backward direction, collide at a predetermined phase of the plasma wake. During this collision, the beating of the injection laser pulses generates a beat wave with a slow phase velocity that kicks a subset of the background plasma electrons which can be trapped and accelerated. A simplified CPI configuration was proposed and analyzed by Fubiani et al. [16, 17, 19] that uses only two laser pulses with parallel polarizations: an intense pump pulse for wakefield generation and a single counterpropagating (or propagating at a finite angle) injection pulse. Injection is the result of the laser beat wave produced when the backward injection pulse collides with the trailing portion of the pump pulse. This configuration has the advantages of being easier to implement in comparison to the three-pulse CPI scheme, and of requiring less intensity in the injection pulse compared to the ponderomotive injection scheme, since injection is the result of the laser beat wave as opposed to the ponderomotive force of a single injection pulse.

In this paper, a negative plasma density gradient is proposed and analyzed as a method for enhancing the electron beam quality in laser injection schemes. If a laser injection scheme is operated close to threshold, electrons will be injected into the region of the wake that is accelerating but defocusing. To have a trapped electron bunch that is both accelerated and focused, it is necessary to shift the bunch forward in phase. This can be accomplished with a downward density ramp. As the density decreases, the plasma wavelength increases, thus a relativistic electron will be shifted forward in phase relative to the wake. This can shift an electron from the defocusing to the focusing region of the accelerating wake. In addition, if injection occurs on the density down-ramp, the trapping can occur more readily since the phase velocity of the wake is lowered on the down ramp. Numerical examples are given based 
on a three-dimensional (3D) particle tracking code for the specific case of the two-pulse CPI method with density gradients.

The remainder of this paper is organized as follows. The general concept of using density down-ramps is discussed in Sec. II. The analytical expressions for the wakefield driven on a density ramp are derived in Sec. III. Section IV presents the numerical results, in which the motion of test particles are tracked in 3D in the analytically specified fields of the laser pulses and the wakes. A discussion of the results is given in Sec. V. Also included is an Appendix that discusses beam loading.

\section{DENSITY DOWN-RAMPS}

A density down-ramp can enhance the number of trapped and focused electrons by two effects: (1) A decrease in density shifts the position of an electron forward in phase with respect to the wakefield and (2) a decrease in density decreases the phase velocity of the wake, thus providing a lower threshold for injection. Consider a change in density from $n_{i}$ to $n_{f}\left(n_{i}>n_{f}\right)$ over a length $L_{t}$ and assume that the electron and laser are all moving in the forward direction $(z)$ with velocity $c$. The phases of the electron before and after the transition are given by $\psi_{i}=k_{p i} \zeta$ and $\psi_{f}=k_{p f} \zeta$, respectively, assuming that the slippage between the electron and the drive laser pulse is small over $L_{t}$ ( $\zeta$ is approximately constant), where $\zeta=z-c t$ is the position of the electron behind the drive pulse $(\zeta<0$ behind the drive pulse), and $k_{p i}=\omega_{p i} / c$ and $k_{p f}=\omega_{p f} / c$ are the plasma wavenumbers evaluated at $n_{i}$ and $n_{f}$, respectively. The change in phase of the electron after the density transition is $\Delta \psi=\psi_{i}-\psi_{f}$, i.e.,

$$
\Delta \psi=\psi_{i}\left[1-\left(n_{f} / n_{i}\right)^{1 / 2}\right] \simeq \psi_{i}\left(\Delta n / 2 n_{i}\right)
$$

assuming $\Delta n=n_{i}-n_{f} \ll n_{i}$. Hence, the change in density required to shift an electron forward in phase by a small amount (e.g., $\Delta \psi \sim \pi / 4)$ is $\Delta n / n_{i}=2\left(\Delta \psi / \psi_{i}\right)=2\left(\Delta \psi / k_{p i} \zeta\right)$. Note that rephasing becomes easier (a smaller $\Delta n / n_{i}$ is required) with increasing distance behind the driver (larger $|\zeta|$ ). Hence, rephasing is more efficient for the three-pulse CPI configuration than for two-pulse CPI, assuming the injection point for three-pulse CPI is behind the first wake period.

If the injection (pulse collision) point was to occur on the down-ramp (as opposed to prior to it), then trapping could be further enhanced due to the decrease in phase velocity 
of the wake on the down-ramp. The wake phase velocity $v_{p}$ can be calculated from the wake phase $\psi=k_{p}(z)(z-c t)$ via $v_{p} / c=-(\partial \psi / \partial c t) /(\partial \psi / \partial z)$. This gives

$$
v_{p} / c=1 /\left(1+k_{p}^{-1} \zeta d k_{p} / d z\right)
$$

where $d k_{p} / d z=\left(k_{p} / 2 n\right) d n / d z$. Since $\zeta<0$ behind the drive pulse, the phase velocity decreases on a density down-ramp $(d n / d z<0)$. Note that this effect becomes more pronounced the larger the distance behind the driver. Thus, the reduction in phase velocity due to the down-ramp is potentially more effective for three-pulse CPI than for two-pulse CPI. Eventually, even in the absence of an injection pulse, the down-ramp leads to wavebreaking and injection for a sufficiently large distance behind the pump pulse [7], assuming that the wake amplitude does not damp.

\section{PLASMA RESPONSE}

The cold fluid equations, describing the evolution of the plasma density $n$, the normalized electron fluid momentum $\mathbf{u}=\mathbf{p} / m c$, the normalized electrostatic potential $\phi=e \Phi / m c^{2}$, and the normalized vector potential $\mathbf{a}=e \mathbf{A} / m c^{2}$, are given by

$$
\begin{aligned}
\partial n / \partial c t+\nabla \cdot(n \mathbf{u} / \gamma) & =0 \\
\partial(\mathbf{u}-\mathbf{a}) / \partial c t & =\nabla(\phi-\gamma) \\
\nabla^{2} \phi & =k_{p 0}^{2}\left[n / n_{00}-n_{0}(z)\right], \\
\left(\nabla^{2}-\partial^{2} / \partial c t^{2}\right) \mathbf{a} & =k_{p 0}^{2}\left(n / n_{00}\right) \mathbf{u} / \gamma+\nabla \partial \phi / \partial c t
\end{aligned}
$$

where $\gamma=\left(1+u^{2}\right)^{1 / 2}, n_{0}(z)$ is the initial density profile, $k_{p 0}=\left(4 \pi n_{00} e^{2} / m c^{2}\right)^{1 / 2}$ is the plasma wavenumber evaluated at the constant density $n_{00}=n_{0}(0)$, and $\nabla \times(\mathbf{u}-\mathbf{a})=0$ has been assumed along with the gauge condition $\nabla \cdot \mathbf{a}=0$.

These equations will be solved order by order with respect to the small parameter $\left|a_{L}\right| \ll$ 1, which is the normalized amplitude of the laser field. To first order, all quantities are small except for $\mathbf{u}_{1} \simeq \mathbf{a}_{L}$. The first order quantities are given by

$$
\begin{aligned}
\partial n_{1} / \partial c t+\nabla \cdot\left(n_{0} \mathbf{u}_{1}\right) & =0 \\
\partial\left(\mathbf{u}_{1}-\mathbf{a}_{L}\right) / \partial c t & =\nabla \phi_{1} \\
\nabla^{2} \phi_{1} & =k_{p 0}^{2} n_{1} / n_{00}
\end{aligned}
$$


which can be combined to yield

$$
\nabla^{2} \partial^{2} \phi_{1} / \partial c t^{2}+\nabla \cdot\left[k_{p}^{2}\left(\nabla \phi_{1}+\partial \mathbf{a}_{L} / \partial c t\right)\right]=0
$$

where $k_{p}^{2}=k_{p 0}^{2} n_{0}(z) / n_{00}$. Since the primary contributions to the first order quantities are on the fast time scale, i.e., $\phi_{1} \sim \exp [i k(z-c t)]$, where $k$ is the laser wavenumber, the first order quantities scale as $\phi_{1} \sim k_{p}^{2} a_{L} /\left(k^{4} r_{0} L_{t}\right), n_{1} / n_{00} \sim a_{L} /\left(k^{2} r_{0} L_{t}\right),\left(u_{1}-a_{L}\right)_{z} \sim k_{p}^{2} a_{L} /\left(k^{4} r_{0} L_{t}\right)$, and $\left(u_{1}-a_{L}\right)_{\perp} \sim k_{p}^{2} a_{L} /\left(k^{5} r_{0}^{2} L_{t}\right)$, where $L_{t}$ is the scale length of the axial density transition, $\partial k_{p}^{2} / \partial z \sim k_{p}^{2} / L_{t}$, and $r_{0}$ is the scale length of the transverse gradient. Since $k / k_{p} \gg 1$, $k L_{t} \gg 1$, and $k r_{0} \gg 1$, all first order quantities will be neglected except for $\mathbf{u}_{1} \simeq \mathbf{a}_{L}$.

To second order,

$$
\begin{aligned}
\partial n_{2} / \partial c t+\nabla \cdot\left(n_{0} \mathbf{u}_{2}\right) & =0 \\
\partial\left(\mathbf{u}_{2}-\mathbf{a}_{2}\right) / \partial c t & =\nabla\left(\phi_{2}-a_{L}^{2} / 2\right), \\
\nabla^{2} \phi_{2} & =k_{p 0}^{2} n_{2} / n_{00}, \\
\left(\nabla^{2}-\partial^{2} / \partial c t^{2}\right) \mathbf{a}_{2} & =k_{p}^{2}(z) \mathbf{u}_{2}+\nabla \partial \phi_{2} / \partial c t,
\end{aligned}
$$

along with $\nabla \cdot \mathbf{a}_{2}=0$. These equations can be combined to yield

$$
\left(\partial^{2} / \partial c t^{2}+k_{p}^{2}\right) \nabla \phi_{2}-k_{p}^{2} \nabla a_{L}^{2} / 2=\left(\nabla^{2}-\partial^{2} / \partial c t^{2}-k_{p}^{2}\right) \partial \mathbf{a}_{2} / \partial c t
$$

In both the uniform plasma limit $\left(k_{p}^{2}=k_{p 0}^{2}\right)$ and the 1D limit $\left(\nabla_{\perp}=0\right), \mathbf{a}_{2}=0$.

The above equation can be solved in the limits $k_{p} L_{t} \gg 1$ and $k_{p} r_{0} \gg 1$ by assuming an ordering $\left|\phi_{2}\right| \gg\left|a_{\perp 2}\right|>\left|a_{z 2}\right|$. As is shown below, these terms scale as $a_{\perp 2} \sim \phi_{2} /\left(k_{p}^{2} r_{0} L_{t}\right)$ and $a_{z 2} \sim \phi_{2} /\left(k_{p}^{3} r_{0}^{2} L_{t}\right)$. This last scaling follows from $\nabla \cdot \mathbf{a}_{2}=0$, i.e., $a_{z 2} \sim a_{\perp 2} /\left(k_{p} r_{0}\right)$. In the following, an averaging over the fast laser frequency is assumed such that the time and axial derivatives scale as $\partial / \partial c t \sim \partial / \partial z \sim k_{p}$. Taking the axial component of the above equation yields to leading order

$$
\left(\frac{\partial^{2}}{\partial c t^{2}}+k_{p}^{2}\right) \frac{\partial \phi_{2}}{\partial z} \simeq k_{p}^{2}(z) \frac{\partial}{\partial z}\left(\frac{a_{L}^{2}}{2}\right)
$$

where a term of order $k_{p}^{3} a_{z 2} \sim \phi_{2} / r_{0}^{2} L_{t}$ has been neglected. This determines the axial wakefield $E_{z} \sim \partial \phi_{2} / \partial z$, neglecting terms of order $1 /\left(k_{p}^{2} r_{0} L_{t}\right)$ or higher. To determine the electromagnetic contribution to the wake $\left(a_{\perp 2}\right)$, the transverse component of Eq. (15) is operated on by $\partial / \partial z$, which yields

$$
\frac{\partial}{\partial z}\left[\left(\nabla^{2}-\frac{\partial^{2}}{\partial c t^{2}}-k_{p}^{2}\right) \frac{\partial a_{x 2}}{\partial c t}\right]=\left(\frac{\partial k_{p}^{2}}{\partial z}\right) \frac{\partial}{\partial x}\left(\phi_{2}-\frac{a_{L}^{2}}{2}\right) .
$$


Scaling the operators in the above equation implies $a_{x 2} \sim \phi_{2} /\left(k_{p}^{2} r_{0} L_{t}\right)$. Hence, the transverse electron field of the wake is given to leading order by $E_{x} \sim \partial \phi_{2} / \partial x$, where terms of order $1 /\left(k_{p} L_{t}\right)$ or higher are neglected.

Consider the wakefields being driven by a forward-going pump laser pulse $(i=0)$ and a backward-going injection laser pulse $(i=1)$, the fields of which are described by the normalized vector potentials $\mathbf{a}_{i}=e \mathbf{A}_{i} / m_{e} c^{2}$. The wake driven by the beating of the pump and injection pulse will be neglected, as discussed in Ref. [16, 17]. The transverse laser field (linearly polarized in the $x$-direction and propagating along the $z$-axis) is given by [20]

$$
a_{x i}\left(r, \zeta_{i}\right)=\hat{a}_{i}\left(r, \zeta_{i}\right) \cos \psi_{i}
$$

with

$$
\hat{a}_{i}\left(r, \zeta_{i}\right)=a_{i}\left(r_{i} / r_{s i}\right) \exp \left(-r^{2} / r_{s i}^{2}\right) \sin \left(\pi \zeta_{i} / L_{i}\right)
$$

for $-L_{i}<\zeta_{i}<0$ and zero otherwise, where $\zeta_{0}=z-\beta_{g 0} c t$ (forward comoving coordinate), $\zeta_{1}=-z-\beta_{g 1} c t$ (backward comoving coordinate), $\beta_{g i}=\eta_{i}$ is the linear group velocity, $\beta_{\phi i}=\eta_{i}^{-1}$ is the linear phase velocity, $\eta_{i}=\left(1-\omega_{p}^{2} / \omega_{i}^{2}-4 / k_{i}^{2} r_{i}^{2}\right)^{1 / 2}$ is the plasma index of refraction, $\psi_{i}=k_{i}\left(z-\beta_{\phi i} c t\right)+\alpha_{i} r^{2} / r_{s i}^{2}+\alpha_{i}-\tan ^{-1} \alpha_{i}$ is the phase, $k_{i}=\omega_{i} /\left(\beta_{\phi i} c\right)$ is the wavenumber, $\omega_{i}$ is the frequency in vacuum, $r_{s i}(z)=r_{i}\left[1+\alpha_{i}(z)\right]^{1 / 2}$ is the spot size, $r_{i}$ is the spot size at waist (here chosen to be $\left.z=Z_{f_{i}}\right), \alpha_{i}(z)=\left(z-Z_{f_{i}}\right)^{2} / Z_{R_{i}}^{2}, Z_{R_{i}}=k_{i} \eta_{i} r_{i}^{2} / 2$ is the Rayleigh length, $L_{i}$ is the pulse length, and a constant has been omitted in the definition of $\psi_{i}$ that represents the initial position and phase of the laser pulse. The axial component of the laser field is specified via $\nabla \cdot \boldsymbol{a}_{i}=0$. Keeping only the leading order contributions gives

$$
a_{z i}\left(r, \zeta_{i}\right) \simeq 2 x\left[\hat{a}_{i}\left(r, \zeta_{i}\right) /\left(k_{i} r_{s i}^{2}\right)\right]\left(\sin \psi_{i}-\alpha_{i} \cos \psi_{i}\right)
$$

Included in the simulations presented below are the wakefields generated by both the pump and injection laser pulses. For linear polarization and assuming $k_{p} r_{0} \gg 1$ and $k_{p} L_{t} \gg$ 1, i.e., a large laser spot and a slowly varying density transition, the normalized electric field of the wakefield $k_{p 0} \mathbf{E}_{i} / E_{0}=-\nabla \phi_{2 i}$ is given by

$$
\left[\partial^{2} / \partial \zeta_{i}^{2}+k_{p}^{2}(z)\right] k_{p 0} \mathbf{E}_{i} / E_{0} \simeq k_{p}^{2}(z) \nabla \hat{a}_{i}^{2} / 4
$$

where $E_{0}=m c^{2} k_{p 0} / e$ is the cold nonrelativistic wavebreaking field evaluated at fixed density $n_{00}$ and the independent variables $\zeta_{i}$ and $z$ have been used. Note that a time-averaging has been performed over the fast laser oscillation (laser frequency), i.e., $\left\langle\hat{a}_{i}^{2} \cos ^{2} \psi_{i}\right\rangle=\hat{a}_{i}^{2} / 2$. The 
axial component of Eq. (21) follows directly from Eq. (16), whereas the transverse component represents the leading order contribution to $E_{\perp}$, neglecting corrections of order $1 /\left(k_{p} L_{t}\right)$ or higher. Assuming that $\hat{a}_{i}^{2}$ is a slowly varying function of $z$, i.e., $\left|\partial \hat{a}_{i}^{2} / \partial \zeta_{i}\right| \gg\left|\partial \hat{a}_{i}^{2} / \partial z\right|$, the solution to Eq. (21) is

$$
\mathbf{E}_{i}\left(r, \zeta_{i}\right) / E_{0}=-\left(k_{p}(z) / 4 k_{p 0}\right) \int_{0}^{\zeta_{i}} d \zeta_{i}^{\prime} \sin \left[k_{p}(z)\left(\zeta_{i}-\zeta_{i}^{\prime}\right)\right]\left(\partial / \partial \zeta_{i}^{\prime}+\nabla_{\perp}\right) \hat{a}_{i}^{2}\left(r, \zeta_{i}^{\prime}\right),
$$

where a term proportional to $\partial \hat{a}_{i}^{2} / \partial z$ has been neglected inside the integrand, which neglects additional terms of order $L_{i} / Z_{R i} \sim 1 /\left(k k_{p} r_{0}^{2}\right) \ll 1$. Specifically, Eq. (21) yields the electric field generated inside the pulse $\left(-L_{i}<\zeta_{i}<0\right)$

$$
\begin{gathered}
\frac{\mathbf{E}_{r i}}{E_{0}}=\frac{a_{i}^{2}}{2} \frac{r_{i}^{2} r}{k_{p 0} r_{s i}^{4}} e^{-2 r^{2} / r_{s i}^{2}}\left[1+\frac{\left(4 \pi^{2} / k_{p}^{2} L_{i}^{2}\right) \cos \left(k_{p} \zeta_{i}\right)-\cos \left(2 \pi \zeta_{i} / L_{i}\right)}{\left(1-4 \pi^{2} / k_{p}^{2} L_{i}^{2}\right)}\right], \\
\frac{\mathbf{E}_{z i}}{E_{0}}=\frac{k_{p}}{k_{p 0}} \frac{a_{i}^{2}}{8} \frac{r_{i}^{2}}{r_{s i}^{2}} e^{-2 r^{2} / r_{s i}^{2}}\left[\frac{\left(4 \pi^{2} / k_{p}^{2} L_{i}^{2}\right) \sin \left(k_{p} \zeta_{i}\right)-\sin \left(2 \pi \zeta_{i} / L_{i}\right)}{\left(1-4 \pi^{2} / k_{p}^{2} L_{i}^{2}\right)}\right],
\end{gathered}
$$

and behind the pulse $\left(\zeta_{i}<-L_{i}\right)$

$$
\begin{gathered}
\frac{\mathbf{E}_{r i}}{E_{0}}=a_{i}^{2} \frac{r_{i}^{2} r}{k_{p 0} r_{s i}^{4}} e^{-2 r^{2} / r_{s i}^{2}}\left(\frac{4 \pi^{2}}{k_{p}^{2} L_{i}^{2}}\right) \frac{\sin \left[k_{p}\left(\zeta_{i}+L_{i} / 2\right)\right] \sin \left(k_{p} L_{i} / 2\right)}{\left(1-4 \pi^{2} / k_{p}^{2} L_{i}^{2}\right)}, \\
\frac{\mathbf{E}_{z i}}{E_{0}}=-\frac{k_{p}}{k_{p 0}} \frac{a_{i}^{2}}{4} \frac{r_{i}^{2}}{r_{s i}^{2}} e^{-2 r^{2} / r_{s i}^{2}}\left(\frac{4 \pi^{2}}{k_{p}^{2} L_{i}^{2}}\right) \frac{\cos \left[k_{p}\left(\zeta_{i}+L_{i} / 2\right)\right] \sin \left(k_{p} L_{i} / 2\right)}{\left(1-4 \pi^{2} / k_{p}^{2} L_{i}^{2}\right)} .
\end{gathered}
$$

For the resonant case $L_{i}=\lambda_{p}$, which corresponds to a condition close to maximum wakefield generation, inside the pulse

$$
\begin{aligned}
& \left.\frac{\mathbf{E}_{r i}}{E_{0}}=\frac{a_{i}^{2}}{2} \frac{r_{i}^{2} r}{k_{p 0} r_{s i}^{4}} e^{-2 r^{2} / r_{s i}^{2}}\left[1-\cos \left(k_{p} \zeta_{i}\right)-\left(k_{p} \zeta_{i} / 2\right) \sin \left(k_{p} \zeta_{i}\right)\right]\right), \\
& \left.\frac{\mathbf{E}_{z i}}{E_{0}}=-\frac{k_{p}}{k_{p 0}} \frac{a_{i}^{2}}{8} \frac{r_{i}^{2}}{r_{s i}^{2}} e^{-2 r^{2} / r_{s i}^{2}}\left[\sin \left(k_{p} \zeta_{i}\right) / 2-\left(k_{p} \zeta_{i} / 2\right) \cos \left(k_{p} \zeta_{i}\right)\right]\right),
\end{aligned}
$$

and behind the pulse,

$$
\begin{gathered}
\frac{\mathbf{E}_{r i}}{E_{0}}=\frac{\pi a_{i}^{2}}{2} \frac{r_{i}^{2} r}{k_{p 0} r_{s i}^{4}} e^{-2 r^{2} / r_{s i}^{2}} \sin \left(k_{p} \zeta_{i}\right), \\
\frac{\mathbf{E}_{z i}}{E_{0}}=-\frac{k_{p}}{k_{p 0}} \frac{\pi a_{i}^{2}}{8} \frac{r_{i}^{2}}{r_{s i}^{2}} e^{-2 r^{2} / r_{s i}^{2}} \cos \left(k_{p} \zeta_{i}\right) .
\end{gathered}
$$

Note that for high laser intensities $\left(\left|a_{i}\right|>1\right)$, this model becomes inaccurate. To describe the nonlinear regime in 3D, as well as other nonlinear effects such as beam loading, requires self-consistent simulations (e.g., particle-in-cell codes), which is beyond the scope of this paper. 


\section{SIMULATION RESULTS}

The effect of density down-ramps on the bunch quality in the colliding pulse injector was studied using a 3D particle tracking code, which evolves the motion of a group of test particles in analytically specified fields. Included in the simulations are the electromagnetic fields of the laser pulses, as determined from the vector potentials given by Eqs. (18)-(20). The wakefields from the pump and injection laser pulses are given by Eqs. (23)-(26). The ion density profile is assumed to be of the form

$$
\frac{n_{0}(z)}{n_{00}}=1-\frac{\tau_{t}}{2}\left[1+\tanh \left(\frac{z-z_{t}}{L_{t}}\right)\right]
$$

where $n_{00}$ is the ion density before the density drop-off $\left(z<z_{t}\right)$, $z_{t}$ is the location of the transition, and $\tau_{t}=\Delta n_{0} / n_{00}$ is the relative change of density.

In the following simulations, the plasma was modeled by a group of test electrons initially at rest and loaded randomly in a three dimensional spatial region of length $\lambda_{p}$ and transverse radius $\lambda_{p} / 2$, uniformly about the $z$-axis, corresponding to a volume $V_{0}=\pi \lambda_{p}^{3} / 4$. This spatial region was chosen to be ahead of the pump laser pulse, and timed with respect to the initial position of the injection pulse such that when the two pulses collide, the test electrons fill the entire region in which trapping may occur. After the collision, various properties of the trapped electron bunch were monitored as function of propagation time, such as the mean energy, the energy spread, the root-mean square (RMS) bunch length, RMS bunch radius, and the trapping fraction. Here, the trapping fraction is defined as $N_{b} / N_{s}$, where $N_{b}$ is the number of test electrons in the bunch and $N_{s}$ the total number of test electrons in the simulation. A quasi-1D configuration with $r_{i} \simeq \lambda_{p}$ was chosen, such that most of the injected electrons, although in a defocusing region of the accelerating wave, will only slowly depart transversely from their initial on-axis location. A density down-ramp will then rephase those electrons onto a trapped and focused orbit. This is shown in Fig. 1, where the cold fluid orbit and trapped and focused orbit are both shown for an initial density $n_{00}$ and another density $30 \%$ smaller. Note that the focusing region has been extended farther behind the pump pulse. The simulations were carried out for normalized laser-plasma parameters $a_{0}=1, \omega_{0} / \omega_{p 0}=50, L_{0}=\lambda_{p 0}$ or $9 \lambda_{p 0} / 8, \omega_{1} / \omega_{p 0}=50$, and $L_{1}=\lambda_{p 0} / 2$. Parameter scans were performed for the injection pulse normalized vector potential $a_{1}$ and for the parameters corresponding to the density ramp such as the length $L_{t}$, the center of the transition $z_{t}$ and the relative change of density $\tau_{t}$. 
Figure 2 shows the resulting electron beam characteristics produced in a uniform plasma without the use of a plasma density gradient $[16,17]$. The total charge in the bunch $Q$ was estimated from the trapping fraction $f_{\text {tr }}$ (the fraction of the initial electrons that remain on trapped and focused orbits) by $Q=e n_{0} f_{\mathrm{tr}} V_{0}$. The bunch density was calculated assuming a square beam profile using the relationships between the length, radius, and corresponding RMS quantities, e.g., $L_{b}=2 \sqrt{3} \sigma_{z}$ is the full beam length, $\sigma_{z}$ the RMS beam length, $r_{b}=2 \sigma_{r}$ the beam radius and $\sigma_{r}$ the beam RMS radius. For the configuration shown in Fig. 2, the typical value of the charge injected is on the order $\sim 4 \mathrm{pC}$. Note also that for high bunch charge, beam loading may become important [see the Appendix for further details]. For a uniform beam profile $n_{b}(r, \zeta)=n_{b} \Theta\left(r_{b}-r\right) \Theta(-\zeta) \Theta\left(\zeta+L_{b}\right)$ of radius $r_{b}$ and length $L_{b}$, where $\Theta$ is a step function, the amplitude of the perturbed density and the axial electric field of the bunch-induced wake is found to be at the back of the bunch [16, 17, 21, 22],

$$
\begin{aligned}
& \delta n / n_{0} \simeq-\left(k_{p} L_{b}\right)^{2}\left(n_{b} / n_{0}\right) / 2 \\
& E_{z} / E_{0} \simeq k_{p} L_{b}\left(n_{b} / n_{0}\right) F_{R}(r)
\end{aligned}
$$

assuming $k_{p} L_{b} \ll 1, \delta n / n_{0} \ll 1$, and $E_{z} / E_{0} \ll 1$, where the radial profile function is $F_{R}(r)=1-k_{p} r_{b} K_{1}\left(k_{p} r_{b}\right) I_{0}\left(k_{p} r\right)$ for $r<r_{b}$. Here $I_{0}$ and $K_{1}$ are modified Bessel functions. For a narrow beam $k_{p}^{2} r_{b}^{2} \ll 1$ and along the axis $F_{R}(r=0) \simeq\left[0.308-0.5 \ln \left(k_{p} r_{b}\right)\right] k_{p}^{2} r_{b}^{2}$. For $k_{p} L_{b}\left(n_{b} / n_{0}\right) \simeq 1$ the linear wake approximation becomes inaccurate and nonlinear methods must be used. Another parameter of interest is a comparison of the wakefield intensity produced by the laser pulse with respect to the wake induced by the beam itself. The latter is required to be much smaller. Using Eq. (30) together with Eq. (33) yield an approximated ratio,

$$
\alpha_{l} \simeq \frac{8}{\pi} \frac{k_{p} L_{b}}{a_{0}^{2}} \frac{n_{b}}{n_{0}} F_{R}(0) \ll 1
$$

which is valid for a laser beam close to the resonant condition $L \simeq \lambda_{p}$. For the case of Fig. 2 beam loading is a negligible effect.

Figure 3 shows the amount of charge in the trapped and focused region of the plasma wave as a function of the density down ramp center $z_{t}$ for the laser-plasma parameters: $a_{0}=1, \omega_{0} / \omega_{p 0}=50, L_{0}=9 \lambda_{p 0} / 8, a_{1}=0.5, \omega_{1} / \omega_{p 0}=50, L_{1}=\lambda_{p 0} / 2, L_{t}=\lambda_{p 0}, \tau_{t}=30 \%$, and $\omega_{p 0} t=147$ after injection. Here $\lambda_{p 0}$ corresponds to the plasma wavelength prior to the density transition. The total charge is increasing temporarily up to a plateau region reached at about $k_{p 0} z_{t}=4 \pi$. As mentioned above, Fig. 1 shows the phase shift of the trapped and 
focused region after passing through the density transition [according to Eq. (1)] as well as a typical orbit of an electron lying in the defocusing region. The latter electrons will circulate along this path towards the high energy region and will cross the extended focusing region of the plasma wave at some later time after injection. Delaying the density transition until those electrons reach the phase $\psi_{f} \simeq 2 \pi$ will allow for rephasing of maximum amount of charge. In Fig. 3, a charge per bunch enhancement by a factor of $\sim 50$ is shown. In this case beam loading may become important, e.g., the bunch induced wakefield $E_{z} / E_{0}$ becomes comparable to the wake generated by the drive laser pulse alone. Nonlinear beam loading will most likely reduce the bunch quality (fraction trapped, average energy, etc.). Note that the oscillation shown in Fig. 3 for the electric field $E_{z} / E_{0}$ as well as for the beam density $k_{p} L_{b}\left(n_{b} / n_{0}\right)$ may be attributed to the extra focusing provided to the rephased electrons by the plasma wave.

Figure 4 plots the parameter $k_{p} L_{b}\left(n_{b} / n_{0}\right)$ (which is used as an indicator for the estimation of the validity of the linear regime in the calculation of beam loading), the electric field $E_{z} / E_{0}$ induced by the electron beam alone and the charge trapped as a function of the injection laser strength $a_{1}$ for the same laser-plasma parameters as of Fig. 3 except for $k_{p 0} z_{t}=12 \pi$. The latter correspond to the region of Fig. 3 were maximum trapping is achieved. Comparing Fig. 2 with Fig. 4 shows a lower trapping threshold as expected $\left(a_{1 \mathrm{~min}} \simeq 0.35\right.$ versus 0.15$)$. The electron beam remains compact as shown in Fig. 5 . The RMS bunch radius $\sigma_{r}$ and RMS bunch duration $\sigma_{z}$ are on the order of a few percent of the plasma wavelength $\lambda_{p 0}$. For a plasma wavelength on the order of $10 \mu \mathrm{m}$, this implies that $<1$ fs (i.e, attosecond scale) bunches can be produced. The bunch normalized emittance is approximated as $\epsilon_{\perp}=\gamma_{0} \beta_{0} \sqrt{\left\langle x^{2}\right\rangle\left\langle x^{\prime 2}\right\rangle} \simeq \sqrt{\left\langle x^{2}\right\rangle\left\langle u_{x}^{2}\right\rangle}$, where $u_{0}=\gamma_{0} \beta_{0} \simeq \gamma_{0}$ is the axial momentum of the electron bunch. For the case of Fig. 5, the emittance is typically small, e.g., $\lambda_{p 0}=40 \mu \mathrm{m}\left(n_{00}=6.9 \times 10^{17} \mathrm{~cm}^{-3}\right)$, implies $\epsilon_{\perp}<0.8 \mathrm{~mm}$.mrad for an average kinetic energy of $\simeq 23 \mathrm{MeV}$. The energy spread $\Delta \gamma / \gamma$ is on the order of a few percent.

A 1D analysis of the dephasing length [1] (which is the typical length required for a trapped electron to outrun the plasma wave, resulting in maximum energy gain) gives $L_{d} \simeq$ $\gamma_{p}^{2} \lambda_{p}$, where $\gamma_{p}=\left(1-\beta_{p}^{2}\right)^{1 / 2}$ is the plasma wave relativistic factor and $\beta_{p} \simeq \beta_{g 0}$ is the plasma wave normalized phase velocity, which is approximately equal to the laser group velocity in the linear regime. For an underdense plasma $\omega_{p} / \omega_{0} \ll 1, \gamma_{p} \simeq \omega_{0} / \omega_{p}$ which corresponds to $L_{d} \sim 10 \mathrm{~cm}$ for $n_{0} \sim 7 \times 10^{17} \mathrm{~cm}^{-3}$. Furthermore, in 3D, the Rayleigh length $Z_{R} \simeq k_{0} r_{0}^{2} / 2$ 
(which is the characteristic distance for laser diffraction) must be compared to the dephasing length and is found to be on the order $4 \mathrm{~cm}$. The beam parameters shown in Fig. 5 are evaluated only after a propagation distance on the order $1 \mathrm{~mm}$. Therefore, the beam quality is expected to improve over longer acceleration distances (increase of kinetic energy, lower energy spread, etc.), up to the limits imposed by dephasing and/or diffraction.

Figure 6(a) plots the parameter $k_{p} L_{b}\left(n_{b} / n_{0}\right)$, bunch charge $Q$, and bunch-induced axial electric field $E_{z} / E_{0}$ as a function of the density transition length $L_{t}$. Figure $6($ a) shows a small effect on the beam quality, e.g., the trapping fraction remains mainly unchanged. The small increase in bunch radius together with the emittance [Fig 6(a)] can be explained by the fact that a long density transition implies that the electron beam remains for a longer period of time in a defocusing phase. The requirement on the transition length $L_{t}$ is to be smaller that the typical distance it takes an electron to outrun the plasma wave. For the laserplasma parameters used in this paper, $Z_{R}<L_{d}$, and $k_{p}^{-1} \ll L_{t} \ll Z_{R}$. This demonstrates the feasibility of using negative plasma density gradients in laboratory experiments as a means for rephasing trapped but unfocused electrons.

Figure 7 shows the trapped bunch charge and corresponding beam loading parameters as a function of the relative change of density $\tau_{t}$ for the laser-plasma parameters $\lambda_{0}=0.8 \mu \mathrm{m}$, $L_{0}=r_{0}=\lambda_{p 0}=40 \mu \mathrm{m}, a_{0}=1, a_{1}=0.2, z_{t}=240 \mu \mathrm{m}, L_{t}=40 \mu \mathrm{m}$, and $c t=147 k_{p 0}^{-1} \simeq$ $935 \mu \mathrm{m}$ after injection. Note that $a_{1}=0.2$ is found to be close to the trapping threshold for $\tau_{t} \simeq 25 \%$, and, by increasing $\tau_{t}$, the trapped charge in the bunch became as high as $Q=20 \mathrm{pC}$ for $\tau_{t}=60 \%$. Consequently, for such values of $\tau_{t}$, the trapping threshold is lower than $a_{1}=0.2$, which is order of magnitude smaller that the laser strength required in ponderomotive injection schemes [11]. Another possible interesting regime would be to lower the drive pulse strength instead of the injection pulse. Using $k_{p} L_{0}=k_{p} r_{0}=k_{p} r_{1}=2 \pi$ along with $a_{1}=0.5$ and $k_{p} L_{1}=4 \pi$ (a length far from the resonant condition in order to minimize the injection wake which could interfere with the wake generated by the drive pulse itself for the case of a modest value of $a_{0}$ ), combined with a long taper length $k_{p} z_{t}>5 \pi$ (to allow maximum injection, e.g., see Fig. 3 ), and $\tau_{t}=30 \%$, may provide a threshold as low as $a_{0}=0.8$. 


\section{CONCLUSION}

Plasma density down-ramps have been proposed as a method for improving electron bunch quality in laser injection schemes. A decrease in density implies an increase in plasma wavelength, which can shift a relativistic electron from the defocusing to the focusing region of the accelerating wakefield. Also, a decrease in density leads to a decrease in wake phase velocity, which can lower the trapping threshold. The specific method of two-pulse CPI was examined using a 3D test particle tracking code. Various properties of the trapped and focused bunch were studied as a function of the ramp and laser parameters. For example, it was found that a density down-ramp of $30 \%$ change in density with $L_{t}=\lambda_{p}$ increased the trapped and focused charge from $0 \mathrm{pC}$ (no ramp) to $25 \mathrm{pC}$ (with ramp) for an injection pulse intensity of $a_{1}=0.3$, and from $<2 \mathrm{pC}$ to $100 \mathrm{pC}$ (which is near the beam loading limit) for $a_{1}=0.5$. Furthermore, no degradation of overall bunch parameters was observed compared to the uniform plasma case. The bunch duration was found to be typically on the order of a few percent of the plasma wavelength, which implies formation of attosecond electron bunches for short plasma wavelengths. The trapped bunch quality was found to depend only weakly on the length of the ramp, indicating that the use of experimentally feasible ramps with $L_{t} \gg \lambda_{p}$ can be effective in enhancing the trapped bunch. Since the use of down ramps increases the number of trapped and focused electrons, the overall trapping threshold for electron injection into the plasma wave is lowered, which allows the production of trapped bunches with lower intensity laser pulses.

One limitation of the approach used in this research is that it relies on test particle simulations in which the fields (lasers and wakes) were specified analytically. Specifically, analytical

expressions were used for the wakefield valid to second order in the normalized laser field $a_{i}^{2}$. This model becomes inaccurate as $a_{i}^{2}$ approaches and exceeds unity and self-consistent simulations, such as using particle-in-cell or fluids codes, are required in this nonlinear regime. A second approximation used in the test particle simulations is the neglection of the wake generated by the trapped bunch, i.e., neglection of beam loading. These test particle simulations indicate that the colliding pulse trapping mechanism is rather robust, i.e., it is easy to trap electrons up to beam loading limit. Again, to fully assess the utility of the colliding pulse injection in the high-charge limit, self-consistent simulations are required.

It should be noted that although this study was restricted to the two-pulse colliding- 
pulse configuration, rephasing and enhancement of the trapped bunch quality by using density transitions is a general method that can be applied to a wide variety of plasma-based accelerators. In general, the relative phase of the bunch in the wake can be repositioned by adjusting the plasma density. As discussed above, the change in density required to shift an electron forward in phase by a small amount is $\Delta n / n_{i}=2\left(\Delta \psi / k_{p i} \zeta\right)$. Note that rephasing becomes easier (a smaller $\Delta n / n_{i}$ is required) with increasing distance behind the driver (larger $|\zeta|)$. Hence, rephasing is more efficient for the bunches trapped in buckets further behind the driver. Typically, only a small change of density is required to shift the phase a significant fraction of a plasma period. Since the wake amplitude is a relatively weak function of density, rephasing can be accomplished by small changes in the density without significantly degrading the accelerating field of the wake. Furthermore, provided that the plasma density transition occurs over a length (or time) that is short compared to the dephasing length of the electron in the wake (or the synchrotron period for a trapped electron in the wake), the rephasing (shifting of the wake relative to the electron) occurs virtually instantaneously with respect to the electron dynamics. Since the dephasing length of a relativistic electron in a plasma wake is relatively long, $L_{d p} \simeq \lambda_{p}^{3} / \lambda$, experimentally producing a density transition with $L_{t} \ll L_{d p}$ is readily achievable, which in turn leads to a near-instantaneous rephasing of the electrons in the wake.

\section{ACKNOWLEDGMENTS}

This work was performed under the auspices the U.S. Department of Energy, Office of High Energy Physics, under contract number DE-AC-03-76SF0098, and by the U.S. Department of Energy SciDAC project, "Advanced Computing for 21st Century Accelerator Science and Technology", which is supported by the Office of High Energy Physics and the Office of Advanced Scientific Computing Research.

\section{APPENDIX A: BEAM LOADING CONSIDERATIONS}

Beam loading, whereby the trapped electron bunch significantly alters the accelerating wakefield, can degrade the quality of the electron bunch. Beam loading is neglected in the particle tracking code. To estimate the effects of beam loading, the wakefield generated by 
the trapped electron bunch propagating in an initially uniform plasma can be calculated and compared to the wakefield driven by the pump laser pulse. Using linear wakefield theory, the normalized density perturbation $\delta n / n_{0} \ll 1$ and normalized axial electric field $E_{z} / E_{0} \ll 1$ driven in an initially uniform plasma by a short electron bunch $\left(n_{b} / n_{0}\right.$ drive term) is given by $[16,17,21,22]$

$$
\begin{aligned}
\left(\frac{\partial^{2}}{\partial \zeta^{2}}+k_{p}^{2}\right) \frac{\delta n}{n_{0}} & =-k_{p}^{2} \frac{n_{b}}{n_{0}}, \\
\left(\nabla_{\perp}^{2}-k_{p}^{2}\right) \frac{E_{z}}{E_{0}} & =-k_{p} \frac{\partial}{\partial \zeta} \frac{\delta n}{n_{0}}
\end{aligned}
$$

where the drive bunch and the resulting wakefields are assumed to be functions of only the variables $\zeta=z-c t$ and $r_{\perp}$. Solving the system of equations (A1)-(A2) for a cylindricallysymmetric drive $n_{b}$ yields

$$
\begin{aligned}
& \frac{\delta n}{n_{0}}=k_{p} \int_{0}^{\zeta} d \zeta^{\prime} \sin \left[k_{p}\left(\zeta-\zeta^{\prime}\right)\right] \frac{n_{b}\left(\zeta^{\prime}\right)}{n_{0}} \\
& \frac{E_{z}}{E_{0}}=k_{p}^{3} \int_{\infty}^{\zeta} d \zeta^{\prime} \int_{0}^{\infty} d r^{\prime} r^{\prime} \cos \left[k_{p}\left(\zeta-\zeta^{\prime}\right)\right] I_{0}\left(k_{p} r_{<}\right) K_{0}\left(k_{p} r_{>}\right) \frac{n_{b}\left(r^{\prime}, \zeta^{\prime}\right)}{n_{0}},
\end{aligned}
$$

where $I_{0}$ and $K_{0}$ are the zeroth-order modified Bessel functions of the second kind, and $r_{<}\left(r_{>}\right)$denote the smaller (larger) of $r$ and $r^{\prime}$ respectively. For a uniform beam profile $n_{b}(r, \zeta)=n_{b} \Theta\left(r_{b}-r\right) \Theta(-\zeta) \Theta(\zeta+L)$ of radius $r_{b}$ and length $L$, where $\Theta$ is a step function, the profile of the perturbed density and the axial wakefield are inside the bunch $-L \leq \zeta \leq 0$

$$
\begin{aligned}
& \delta n / n_{0}=-2\left(n_{b} / n_{0}\right) \sin ^{2}\left(k_{p} \zeta / 2\right) \\
& E_{z} / E_{0}=-\left(n_{b} / n_{0}\right) F_{R}(r) \sin k_{p} \zeta
\end{aligned}
$$

and behind $\zeta<-L$

$$
\begin{aligned}
\delta n / n_{0} & =-2\left(n_{b} / n_{0}\right) \sin \left(k_{p} L / 2\right) \sin k_{p}(\zeta+L / 2), \\
E_{z} / E_{0} & =-\left(n_{b} / n_{0}\right) F_{R}(r)\left[\sin k_{p} \zeta-\sin k_{p}(\zeta-L)\right],
\end{aligned}
$$

where the radial profile function is

$$
F_{R}(r)= \begin{cases}1-k_{p} r_{b} K_{1}\left(k_{p} r_{b}\right) I_{0}\left(k_{p} r\right), & \text { for } r<r_{b} \\ k_{p} r_{b} I_{1}\left(k_{p} r_{b}\right) K_{0}\left(k_{p} r\right), & \text { for } r>r_{b}\end{cases}
$$


with $I_{1}$ and $K_{1}$ the first-order modified Bessel functions. Assuming $k_{p} L \ll 1$ yields at the back of the bunch,

$$
\begin{aligned}
& \delta n / n_{0} \simeq-\left(k_{p} L\right)^{2}\left(n_{b} / n_{0}\right) / 2, \\
& E_{z} / E_{0} \simeq k_{p} L\left(n_{b} / n_{0}\right) F_{R}(r) .
\end{aligned}
$$




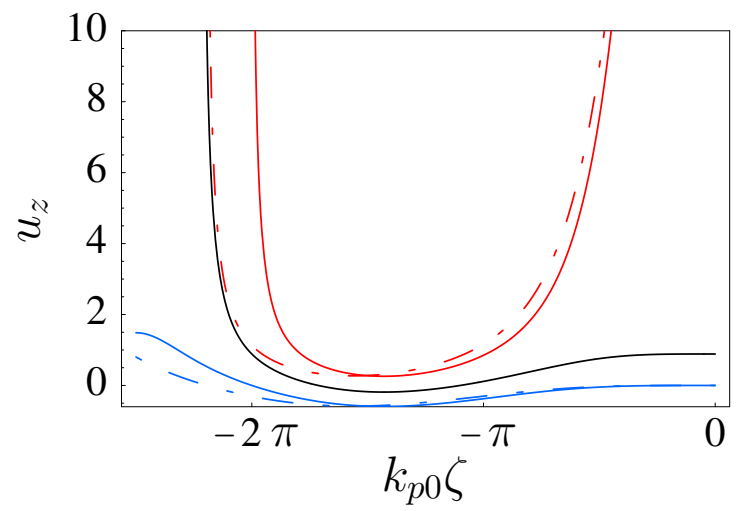

FIG. 1: (Color) Phase space plot showing cold fluid orbit for $n_{0} / n_{00}=1$ (blue solid line), $n_{0} / n_{00}=$ 0.7 (blue dot-dashed line), trapped and focused orbit for $n_{0} / n_{00}=1$ (red solid line), $n_{0} / n_{00}=0.7$ (red dot-dashed line), and orbit of an electron in a trapped but defocusing region of the wakefield for $n_{0} / n_{00}=1$ (black solid line), with laser parameters: $L_{0}=\lambda_{p 0}$ and $a_{0}=1$.

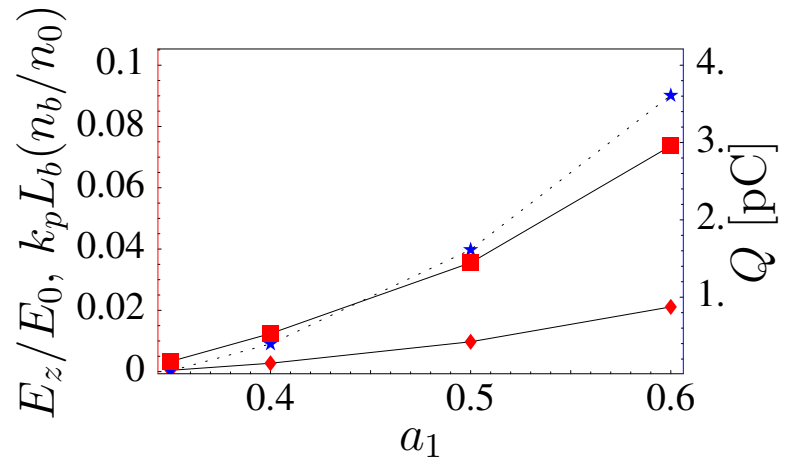

FIG. 2: (Color) Bunch charge $Q$ in pC (right vertical axis, stars), $k_{p} L_{b}\left(n_{b} / n_{0}\right)$ (left vertical axis, squares), and normalized axial electric field $E_{z} / E_{0}$ (left vertical axis, points) generated by the electron bunch alone (here the laser contribution is not included) versus $a_{1}$ with $\lambda_{0}=0.8 \mu \mathrm{m}$, $L_{0}=9 \lambda_{p 0} / 4, r_{0}=\lambda_{p 0}=40 \mu \mathrm{m}, a_{0}=1$, homogeneous plasma, e.g., no density ramp and $c t=47 k_{p 0}^{-1} \simeq 300 \mu \mathrm{m}$ after injection. 


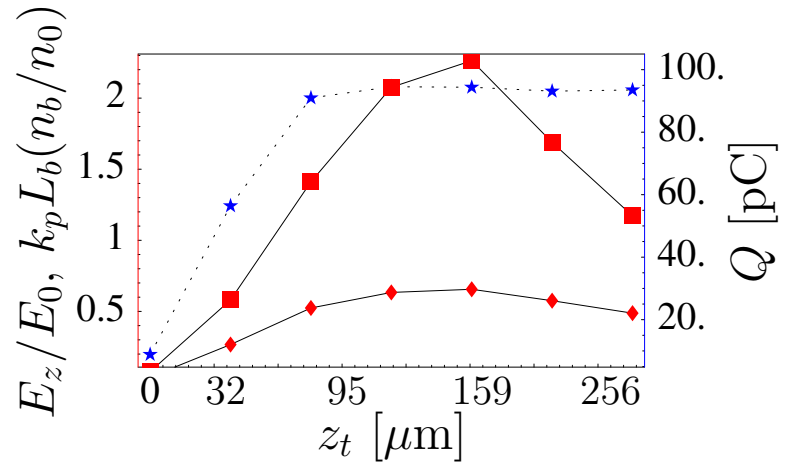

FIG. 3: (Color) Bunch charge $Q$ in pC (right vertical axis, stars), $k_{p} L_{b}\left(n_{b} / n_{0}\right)$ (left vertical axis, squares), and normalized axial electric field $E_{z} / E_{0}$ (left vertical axis, points) generated by the electron bunch alone versus $z_{t}$ with $\lambda_{0}=0.8 \mu \mathrm{m}, L_{0}=9 \lambda_{p 0} / 4, r_{0}=\lambda_{p 0}=40 \mu \mathrm{m}, a_{0}=1, a_{1}=0.5$, $L_{t}=\lambda_{p 0}, \tau_{t}=30 \%$ and $c t=147 k_{p 0}^{-1} \simeq 935 \mu \mathrm{m}$ after injection.

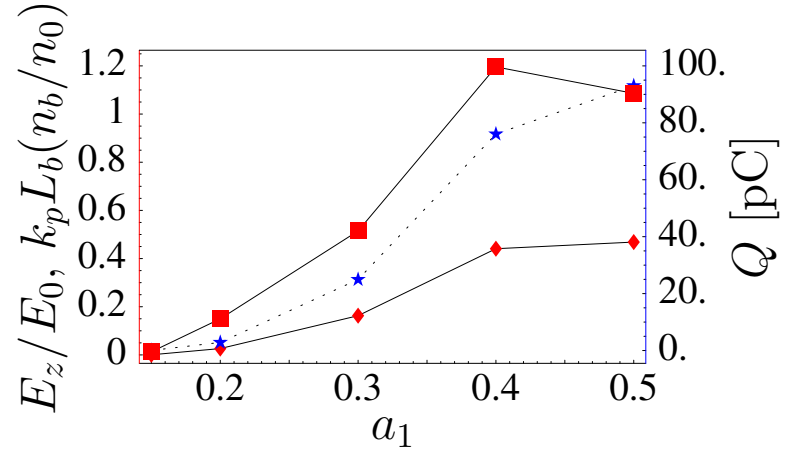

FIG. 4: (Color) Bunch charge $Q$ in pC (right vertical axis, stars), $k_{p} L_{b}\left(n_{b} / n_{0}\right)$ (left vertical axis, squares) and normalized axial electric field $E_{z} / E_{0}$ (left vertical axis, points) generated by the electron bunch alone versus $a_{1}$ with $\lambda_{0}=0.8 \mu \mathrm{m}, L_{0}=9 \lambda_{p 0} / 4, r_{0}=\lambda_{p 0}=40 \mu \mathrm{m}, a_{0}=1$, $z_{t}=240 \mu \mathrm{m}, L_{t}=\lambda_{p 0}, \tau_{t}=30 \%$, and $c t=147 k_{p 0}^{-1} \simeq 935 \mu \mathrm{m}$ after injection. 


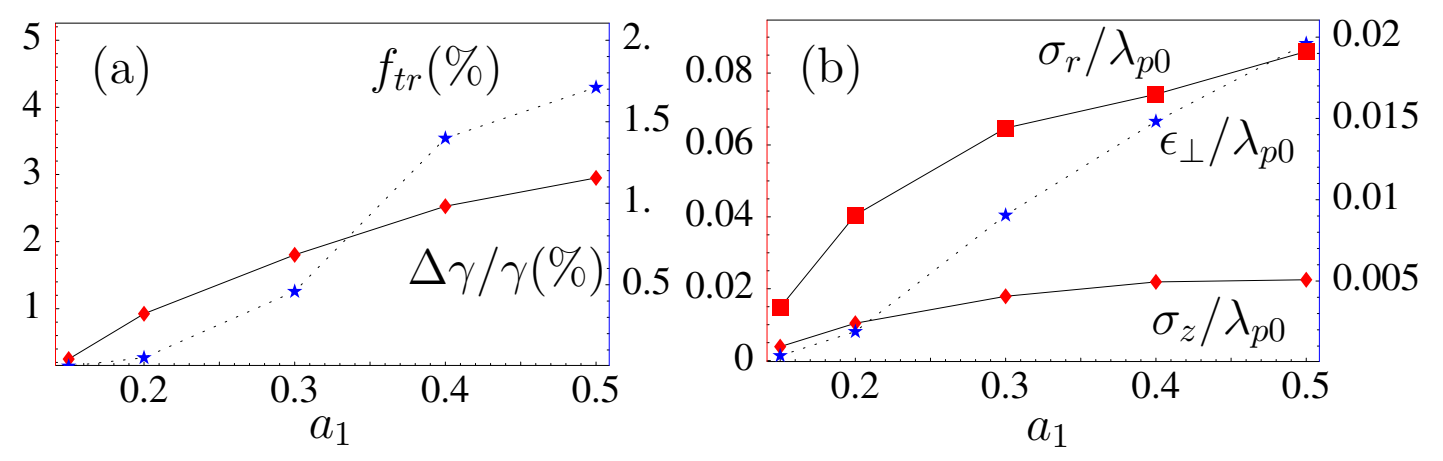

FIG. 5: (Color) Trapped bunch parameters versus $a_{1}$ (for two collinear, counterpropagating laser pulses with equal polarization, $a_{0}=1, \omega_{0} / \omega_{p 0}=50, L_{0}=9 \lambda_{p 0} / 8, \omega_{1} / \omega_{p 0}=50, L_{1}=\lambda_{p 0} / 2$, $k_{p 0} z_{t}=12 \pi, k_{p 0} L_{t}=2 \pi, \tau_{t}=30 \%$, and $c t=147 k_{p 0}^{-1}$ ). (a) Trapping fraction $f_{\text {tr }}$ (right vertical axis) and relative energy spread $\Delta \gamma / \gamma$ (left vertical axis). (b) Bunch length $\sigma_{z} / \lambda_{p 0}$ (left vertical axis), RMS radius $\sigma_{r} / \lambda_{p 0}$ (left vertical axis), and normalized transverse RMS emittance $\epsilon_{\perp} / \lambda_{p 0}$ (right vertical axis).
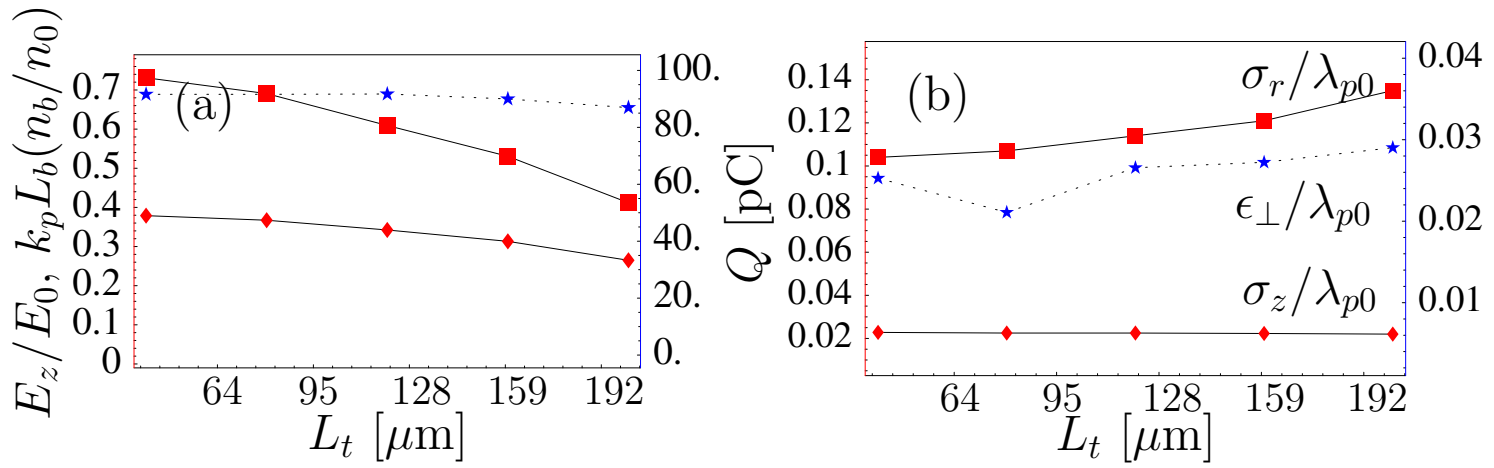

FIG. 6: (Color) (a) Bunch charge $Q$ in pC (right vertical axis, stars), $k_{p} L_{b}\left(n_{b} / n_{0}\right)$ (left vertical axis, squares), and normalized axial electric field $E_{z} / E_{0}$ (left vertical axis, points) generated by the electron bunch alone. (b) Bunch length $\sigma_{z} / \lambda_{p 0}$ (left vertical axis), RMS radius $\sigma_{r} / \lambda_{p 0}$ (left vertical axis), and normalized transverse RMS emittance $\epsilon_{\perp} / \lambda_{p 0}$ (right vertical axis) versus $L_{t}$ for the laser-plasma parameters: $\lambda_{0}=0.8 \mu \mathrm{m}, L_{0}=9 \lambda_{p 0} / 4, r_{0}=\lambda_{p 0}=40 \mu \mathrm{m}, a_{0}=1, a_{1}=0.5$, $z_{t}=280 \mu \mathrm{m}, \tau_{t}=30 \%$ and $c t=147 k_{p 0}^{-1} \simeq 935 \mu \mathrm{m}$ after injection. 


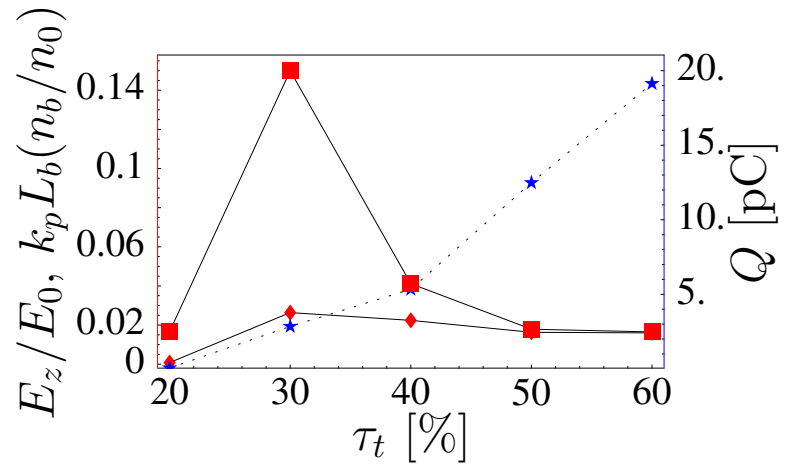

FIG. 7: (Color) Bunch charge $Q$ in pC (right vertical axis, stars), $k_{p} L_{b}\left(n_{b} / n_{0}\right)$ (left vertical axis, squares) and normalized axial electric field $E_{z} / E_{0}$ (left vertical axis, points) generated by the electron bunch alone versus $\tau_{t}$ with $\lambda_{0}=0.8 \mu \mathrm{m}, L_{0}=r_{0}=\lambda_{p 0}=40 \mu \mathrm{m}, a_{0}=1, a_{1}=0.2$, $z_{t}=240 \mu \mathrm{m}, L_{t}=40 \mu \mathrm{m}$, and $c t=147 k_{p 0}^{-1} \simeq 935 \mu \mathrm{m}$ after injection. 
[1] E. Esarey, P. Sprangle, J. Krall, and A. Ting, IEEE Trans. Plasma Sci. 24, 252 (1996).

[2] K.-C. Tzeng, W. B. Mori, and T. Katsouleas, Phys. Rev. Lett. 79, 5258 (1997).

[3] E. Esarey, B. Hafizi, R. Hubbard, and A. Ting, Phys. Rev. Lett. 80, 5552 (1998).

[4] A. Pukhov and J. Meyer-ter-Vehn, Appl. Phys. B 74, 355 (2002).

[5] F. S. Tsung, R. Narang, W. B. Mori, C. Joshi, R. A. Fonseca, and L. O. Silva, Phys. Rev. Lett. 93, 185002 (2004).

[6] S. V. Bulanov, F. Pegoraro, A. M. Pukhov, and A. S. Sakharov, Phys. Rev. Lett. 78, 4205 (1997).

[7] S. Bulanov, N. Naumova, F. Pegoraro, and J. Sakai, Phys. Rev. E 58, R5257 (1998).

[8] P. Tomassini, M. Galimberti, A. Giulietti, D. Giulietti, L. A. Gizzi, L. Labate, and F. Pegoraro, Phys. Rev. ST Accel. Beams 6, 121301 (2003).

[9] H. Suk, N. Barov, J. B. Rosenzweig, and E. Esarey, Phys. Rev. Lett. 86, 1011 (2001).

[10] C. Geddes, C. Toth, J. van Tilborg, E. Esarey, C. Schroeder, D. Bruhwiler, C. Nieter, J. Cary, and W. Leemans, Nature 431, 538 (2004).

[11] D. Umstadter, J. K. Kim, and E. Dodd, Phys. Rev. Lett. 76, 2073 (1996).

[12] R. G. Hemker, K.-C. Tzeng, W. B. Mori, C. E. Clayton, and T. Katsouleas, Phys. Rev. E 57, 5920 (1998).

[13] J. R. Cary, R. E. Giacone, C. Nieter, and D. L. Bruhwiler, Phys. Plasmas 12, 056704 (2005).

[14] E. Esarey, R. F. Hubbard, W. P. Leemans, A. Ting, and P. Sprangle, Phys. Rev. Lett. 79, $2682(1997)$.

[15] C. B. Schroeder, P. B. Lee, J. S. Wurtele, E. Esarey, and W. P. Leemans, Phys. Rev. E 59, 6037 (1999).

[16] G. Fubiani, E. Esarey, C. B. Schroeder, and W. P. Leemans, Phys. Rev. E 70, 016402 (2004).

[17] G. Fubiani, Ph.D. thesis, Lawrence Berkeley National Laboratory \& University Paris XI, Orsay (2005), LBNL-report \# 57514.

[18] H. Kotaki, S. Masuda, M. Kando, J. K. Koga, and K. Nakajima, Phys. Plasmas 11, 3296 (2004).

[19] E. Esarey, G. Fubiani, C. Schroeder, B. Shadwick, W. Leemans, J. Cary, and R. Giaconne, Bull. Am. Phys. Soc. 47, 280 (2002). 
[20] E. Esarey and W. P. Leemans, Phys. Rev. E 59, 1082 (1999).

[21] R. Keinings and M. E. Jones, Phys. Fluids 30, 252 (1987).

[22] T. Katsouleas, S. Wilks, P. Chen, J. M. Dawson, and J. J. Su, in Part. Accel. Conf. (1987), vol. 22 , pp. $81-99$. 